\title{
Multidisciplinary Research Efforts in Post-Earthquake Civil Infrastructure Reconnaissance
}

\section{Mr. Jack Bergquist, California Polytechnic State University, San Luis Obispo}

Jack Bergquist is a undergraduate student at California Polytechnic State University - San Luis Obispo with a major in architectural engineering and minor in Italian. He has formally been engaged in earthquake engineering and reconnaissance research for the past year, and has a specific interest in the seismic performance of historic and vernacular structures. After graduation, he plans to continue working in this field as a structural engineer for a non-profit organization focusing on building development and disaster recovery in developing countries.

\section{Dr. Anahid Behrouzi, California Polytechnic State University, San Luis Obispo}

Anahid Behrouzi is an assistant professor of architectural engineering at California Polytechnic State University - San Luis Obispo. She has been involved with STEM education beginning in 2003 as a volunteer and summer instructor with the North Carolina Museum of Life and Science. She has been engaged with undergraduate/graduate course delivery in the topic areas of engineering problem-solving, structural engineering, and reinforced concrete design at North Carolina State University (2008-2011), the University of Illinois at Urbana-Champaign (2012-2015), Tufts University (2015-2016), and Cal Poly - SLO (2016-present). She has a BS in civil engineering and BA in Spanish language \& literature from North Carolina State University, and a MS/PhD in civil engineering from the University of Illinois at Urbana-Champaign. 


\title{
Multidisciplinary Research Efforts in Post-Earthquake Civil Infrastructure Reconnaissance
}

\begin{abstract}
:
To address existing challenges with filtering and classification of post-earthquake structural damage images, the authors are engaged in a multidisciplinary project to develop and train a machine-learning algorithm that identifies relevant photographs and assigns damage tags to those images. The research team is predominantly comprised of undergraduate students and is led by a structural engineering and a computer science faculty. While machine-learning algorithms have been successfully used for image tagging in a variety of fields (health care, manufacturing, etc.), the extension of this approach for earthquake reconnaissance is only just beginning. As such, the creation and development of this tool is a new and dynamic project-based learning experience for both the students and faculty involved.

This collaborative project emphasizes student initiative and innovation where they are active in all development stages of the tool ranging from collection and tagging of earthquake damage images, coding and testing the machine-learning algorithm, to writing papers for and presenting at conferences. In addition, the unique nature of this project exposes students to a field and possible career path they may not have encountered in their typical course of study. The authors provide a comprehensive discussion of the results of faculty and student surveys/ interviews and conclude by highlighting some of the greatest benefits of the multidisciplinary project. They also point out lessons learned engaging in a project with a large scope, diverse experts (who have limited knowledge of the partnering disciplines), and a number of undergraduate students who began as novices in their respective research area.
\end{abstract}

\section{Introduction:}

The multidisciplinary project presented in this paper brings together the fields of structural engineering and computer science to address an existing shortcoming in seismic reconnaissance. Presently, expert engineers are required to manually filter and tag post-earthquake images of damaged civil infrastructure (acquired from engineering inspection teams or other formal/social media platforms); the collaborative research team is attempting to automate these time intensive and technically challenging tasks by developing a robust deep learning (DL) algorithm.

The research team is based out of California Polytechnic State University - San Luis Obispo, a predominantly undergraduate public university located on the West Coast. As a reflection of this academic environment, the research team is made up of two junior tenure-track faculty members from the departments of architectural (structural) engineering and computer science. The faculty advisors collaborate to set overarching goals and outcomes of the project, but more or less, independently lead a team of students in their respective fields. The teaching institution advocates applied learning opportunities that promote student initiative and self-efficacy. As a result, students are involved in project development including presenting suggestions for deliverables and participating in research dissemination. 
This paper focuses on the educational outcomes of the multidisciplinary research. Specifically, it summarizes the research roles, learning gains, and unique opportunities afforded to each research team member (students and faculty) based on data gathered during detailed interviews/surveys, and includes discussions of how:

- Multidisciplinary work has affected their understanding of engineering challenges in their respective field and of complementary fields;

- Peer-teaching across disciplines has broadened their existing technical knowledge;

- Skills acquired during this experience has strengthened problem-solving abilities in relevant areas to future research/industry work;

- Authorship and attendance of conferences has contributed to the extension of their professional network, new research ideas, or future career goals;

- Prevalence of undergraduate students on the project influences the research team structure and progress; and

- Interactions on this multidisciplinary team have challenged them to overcome differences in knowledge background and skills to successfully address a common research goal.

\section{Project Description/Objectives:}

The ultimate outcome of the project is to develop a deep learning (DL) algorithm to automate the process of filtering and classifying images of damaged civil infrastructure collected after an earthquake event. The training images are gathered from existing databases of previous events, inspections conducted by professional engineers, or various formal/social media platforms (specific sources include NISEE PEER library [1]; EERI Learning from Earthquakes Reconnaissance Archive [2]; GEER Reconnaissance Reports [3]; datacenterhub.org [4]). A minimum of 200 clear, high-resolution images were assembled for each damage-structure pair of interest and individually tagged by the architectural engineering team using a software tool developed by the computer science team that outputs .xml files, input to the DL algorithm [5].

The computer science team facilitated the training and validation of the DL algorithm. These efforts have already shown promise with respect to: (i) binning building images as damage-no damage ( $88 \%$ accuracy) with an average time of 0.2 seconds/image, (ii) drawing a bounding box around damage in buildings (85\% accuracy) and short/captive reinforced concrete columns with shear damage (77\% accuracy), (iii) identifying roadway images exhibiting cracking of varying directionality and severities (92\% accuracy) and railway images affected by horizontal offset/lateral translation (80\% accuracy) [6], [7].

At the onset of the project, the research team hoped to develop a robust set of DL algorithms that will be made publically available to enable rapid identification and tagging of a wide array of building/infrastructure damage in the immediate aftermath of an earthquake. This real-time response could assist with emergency response and repair/recovery decision-making. There are also many existing repositories of earthquake reconnaissance images with limited metadata on damage that appear in the images, in the longer timeframe the DL algorithms could be used to add this metadata which would assist engineers to conduct analyses of damage in a particular earthquake and compare to outcomes of other geographically or chronologically diverse seismic events. Another objective of the research team is to collaborate with other researchers to develop a database similar to ImageNet in utility, but is focused on the needs of the seismic 
reconnaissance community. ImageNet is a premier database with over 14 million images that have been classified and are used by the computer science machine-learning community to train and validate image classification algorithms [8]. Even a modest, but well-curated, image database would be of great benefit to future work in automating image tagging in the earthquake engineering arena.

Over time, additional project goals have been developed by the research team. Largely these result from discussions with industry representatives and private consulting engineers who have taken an interest in the project. For example, the research team is currently working to develop infrastructure maps that will take the tagged damage images from the DL algorithm and use the image's GPS coordinates to place pin locators on infrastructure maps. With the maps it will be possible to update municipalities in real-time after a hazard event to identify damaged buildings/ infrastructure or blocked lifelines (i.e. a landslide covering a roadway) and provide information on the severity of the damage. The maps are also relevant to assist for more involved analyses, again among geographically and chronologically diverse earthquake events.

\section{Composition of the Research Team:}

The faculty team first assembled in October 2016 to begin preliminary investigative work around the topic and writing grant proposals to support student engagement on the project. Recruitment of students for the project began in early December 2016 and at present there are two active undergraduate students from each of the computer science (CS) and structural engineering (SE) fields. Within the last academic quarter, four graduate students have joined the research team (1 CS, $3 \mathrm{SE}$ ); however, this paper will focus on the perspective of students who have been on the project for at least one academic quarter.

Figure 1 illustrates the research team structure and pseudonym assigned for each member on the project. Some basic information on each of the surveyed/interviewed students is listed below:

- $\quad$ SE Student 1 (First Author of Paper):

- Senior student who joined the research team in April 2017.

- Primary tasks: collection/tagging of structural damage images; development of structural damage identification training documents; preparation and presentation of research progress at the Fall 2017 American Concrete Institute (ACI) convention.

- $\quad$ SE Student 2 (Interviewed):

- Senior student who joined the research team in late September 2017.

- Primary tasks: collection/tagging of structural damage images; earthquake reconnaissance in Mexico City in late October 2017 and associated data analysis.

- SE Student 3 (Interviewed):

- Senior student who participated in team early January - June 2017.

- Primary tasks: collection/tagging of structural damage images; development of structural damage identification training documents.

- $\quad$ CS Student $1 \& 2$ (Surveyed):

- Senior students who joined the research team in mid-December 2016.

- Primary tasks: development of deep learning algorithm and associated graphic user interface that allows non-CS experts to bin/classify structural damage images. 


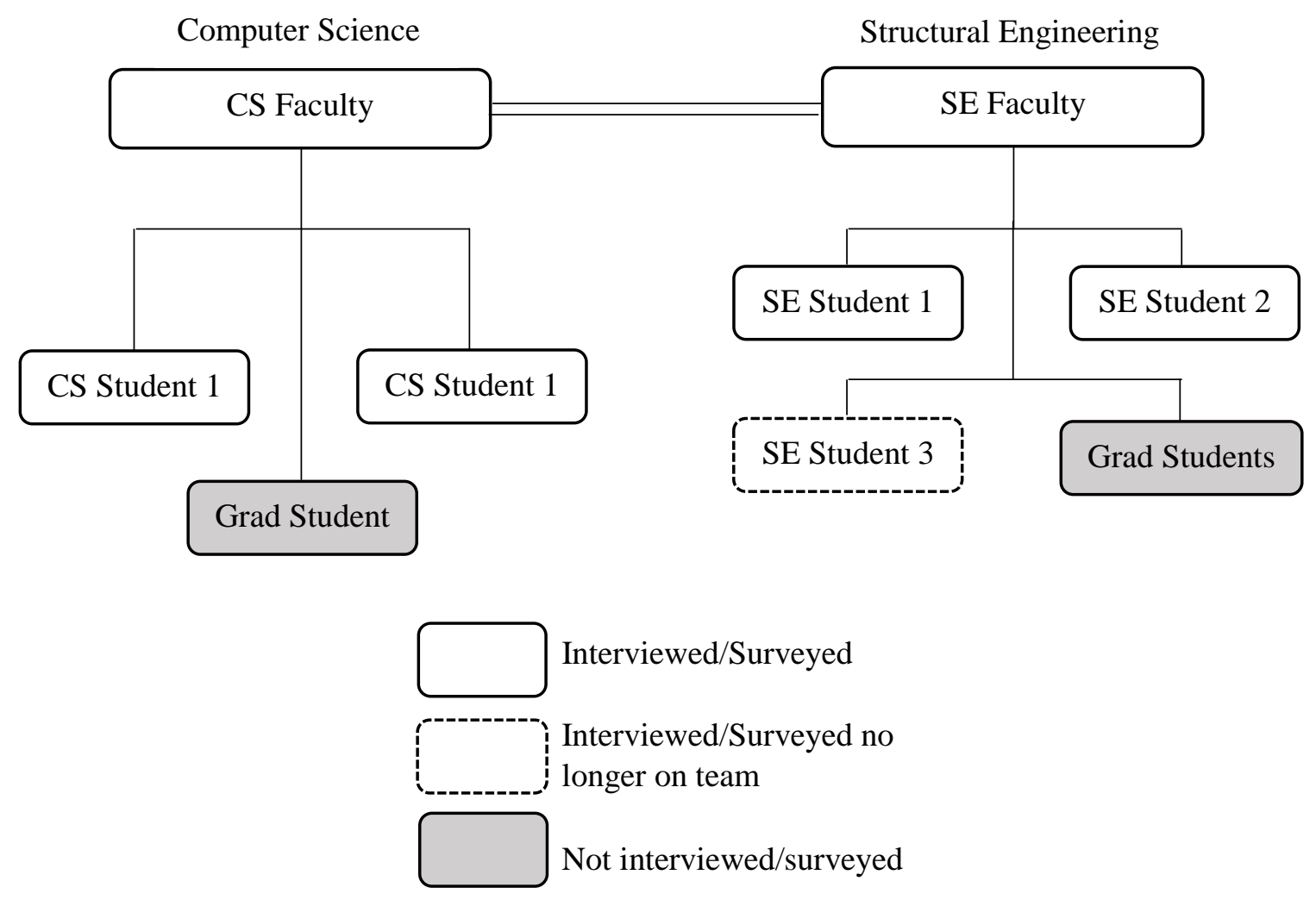

Figure 1: Research Team Structure

Intended Contributions and Learning Gains of Student Team Members:

The SE students are not typically exposed to the humanitarian aspect of structural engineering through their coursework (emergency response/recovery; reconnaissance efforts to inform modifications to building codes). In addition, their coursework includes limited computer coding, primarily in MATLAB and nothing to the degree the CS students experience. Participating in this multidisciplinary project with the CS team, the SE students gain insight into the application of a robust machine-learning image classification algorithm to achieve largely humanitarian goals. Additionally, the SE students develop in engineering fields outside the classical design approaches they are exposed to in traditional coursework.

The CS students have the unique opportunity to assist in creating an algorithm that has real implication in the field of earthquake engineering. The SE team's collaboration provides much needed expertise on the existing problem which informs CS student's DL algorithm solution. Use of machine-learning approaches is becoming pervasive in the computer science field, and as such the CS students gain valuable experience working on a project that will be similar to work they will see in their professional careers. 


\section{Discussion of Assessment Approach and Results:}

To assess educational outcomes of this multidisciplinary research, project members either completed an emailed survey or were interviewed in person. The survey/interview questions were broken down into categories of: cross-discipline understanding, communication, project progress/outcomes, and team structure. The project members, aside from SE Student 1 who is the first author of this paper, were not informed of others' responses and their answers should therefore reflect only their personal experience on the research team.

\section{Faculty Perspective:}

Both faculty advisors responded to an identical set of questions found in Appendix A and were administered via the faculty's elected mode of preference (CS Faculty completed email survey, SE Faculty completed in-person interview). The following section of the paper organizes faculty responses into three primary topic areas: (i) comparison of this multidisciplinary research project versus traditional upper-division courses/projects offered in their respective academic departments, (ii) project progress/timeline, and (iii) communication. The main objective is to identify the successes and lessons learned from pursuing a multidisciplinary project that is in a completely new research area and has required multiple iterations of defining the research problem and project deliverables.

While the primary engineering outcome for the multidisciplinary research is a DL algorithm to facilitate post-earthquake reconnaissance work, the faculty advisors view this as a vehicle for their students to learn and develop as engineers. For this reason, the faculty are active in tracking the quality of specific deliverables to have evidence of their students' learning, regardless of whether these students are paid research assistants (via an internal university grant associated with the project) and/or applying this work towards their senior project course credit.

\section{Multidisciplinary Research Project vs. Traditional Upper-Division Courses/Projects}

The two faculty have differing assessments of the level of multidisciplinary experiences that their students are already exposed to in their respective departments: the CS Faculty believes her department provides plenty of multidisciplinary experiences in traditional coursework, whereas the SE faculty finds her department focuses on multidisciplinary projects at the bookends of a student's college career. Both CS and SE Faculty agree that a multidisciplinary research projects provide students with unique experiences in real engineering problems, echoing findings of an undergraduate multidisciplinary project on image compression that was integrated into a mathematics course at the West Virginia Institute of Technology [9].

\section{CS Faculty:}

The CS Faculty indicates that her department commonly has interdisciplinary upper-division undergraduate coursework and senior design projects. She is already satisfied that students are sufficiently exposed to applied engineering and industry opportunities in different disciplines during the normal course of study. She points out that the variety and richness of CS students' past experiences lends well to the teaming and self-efficacy required on the research team. Therefore, her primary motivation for engaging students on this project is to specifically equip 
them to create and train robust DL algorithms. These types of algorithms are commonplace in many major fields including medical diagnosis, automated car inspection, and facial recognition [10], [11], [12]. More closely related to post-earthquake assessment, DL algorithms have been used with to classify damage patterns in aerial or on-the-ground images of structures; also, photogrammetric approaches have been used to determine interstory drift in tall buildings [13],[14],[15]. The prevalence and novelty of DL algorithms is a draw for the CS students to the research team.

SE Faculty:

The SE Faculty describes upper-division courses in her department as having a deep laser-like focus on structural analysis and design. Aside from the freshman year which consists of architecture studios, the senior capstone project is often the only other opportunity for students to interact with disciplines housed in the College (construction management, architecture, landscape architecture, as well as city and regional planning). Her motivation is to expose students to a new flavor of interdisciplinary work with CS collaborators and to earthquake reconnaissance topics that are almost never broached in traditional SE coursework, but can have significant industry/ humanitarian impact. She sees this research work as a means for students to engage with the source documents of historic or recent hazard events, and to expand their professional network through people they meet via conference attendance and in-field reconnaissance.

\section{Project Progress/Timeline}

This project is technically complex and demands an expert-level knowledge in order to ensure progress. As a result, both the CS and SE Faculty indicate they spend more time on technical tasks in the predominantly undergraduate setting than they would in a research institution. In a multidisciplinary research project at the Rochester Institute of Technology involving robotics and engineering, the senior graduate team members supervise a group of novice students while still playing a critical technical research role [16]. In a project similar to that of this research teams, identifying post-earthquake structural damage in photographs, a Purdue University faculty is leading a post-doctoral student (with expertise in civil engineering and computer science) in creating and implementing a DL algorithm [15]. For the research project described in this paper, there are no senior graduate or post-doctoral students that can function as both a supervisor to younger students and a reliable/knowledgeable work engine. Thus, the faculty - with all their other teaching, research, and service commitments - fulfill this role.

\section{CS Faculty:}

Training a DL algorithm is complex and requires work at a level that goes beyond most undergraduate students' abilities. As such, the CS Faculty has been working independently to complete tasks related to DL algorithm coding and parallel programming to both classify images of structural damage, and place a bounding box around each location of damage. Her students have taken on a simplified, but still challenging, version of the problem where they bin structural damage images into different categories. The CS Faculty strongly believes that her students have worked at a level of efficiency, professionalism, and technical skill that is commensurate with an advanced graduate student, and have made significant contributions to project objectives. There have been obvious learning gains from the student's progress thus far, but concrete technical 
progress on the DL algorithm is affected by the CS Faculty's availability. Her contribution to the project is core to the functionality of the DL algorithm, more so than her students, and her time schedule has been dictates the speed of progress.

SE Faculty:

The SE Faculty has had to take on tasks for which the undergraduate student is not prepared, such as the collection of image sets that relate to identification of complex structural damage types or failures. This time has allowed for SE student learning and now they have reached a stage where the SE Faculty no longer has to conduct filtering/tagging tasks herself; consequently, team efficiency has improved considerably.

Dissemination of research progress via professional conferences has garnered industry support from experienced design engineers and reconnaissance team members. Their input has led to modifications in scope and deliverables to better meet the needs of stakeholders that the faculty did not initially consider in their original project proposal. Despite this being a positive thing, the SE Faculty describes how the dynamic nature of this project has posed a challenge for her and her students where shifting intermediate goals and student turnover has led to a lack of consistency in work quality and output. For students engaged in the research on pay (rather than for course credit) the SE Faculty is flexible in allowing them to choose when they want to work or leave the research team altogether. She has resolved to maintain flexible deliverables, but in a manner, that better facilitates and tracks students' growth while contributing to the project.

\section{Communication}

CS and SE Faculty have both identified lack of communication (or miscommunication) as an issue that has impeded progress on the project. Varying degrees of understanding of desired deliverables have caused challenges in handovers of desired data for training the DL algorithm and resulted in project delays. A study done on 62 different scientific collaborations supported by a program of the National Science Foundation (NSF) in 1998-1999 found that barriers in communication have been alleviated by having a consistent time to meet and communicate with your collaborator [17]. In early 2018, the CS and SE Faculty have begun having more consistent weekly meetings to discuss objectives and outcomes, and to this point, have found this has led to more rapid intermediate deliverable turn-around from both teams and less overall stress associated with the project.

CS Faculty:

The CS Faculty indicates that one communication issue has negatively impacted project progress of her and her students: delays in receiving complete filtered tagged image sets from the structural engineering team. As a result the CS students have had to independently find data with which to train the DL algorithm. Miscommunication and under-communication has become a point of contrition between the SE and CS team. The CS Faculty stated that to ensure efficiency of the project there must be frequent messages and accurate status reports, and moving forward both faculty have agreed to consistent weekly meetings. In future multidisciplinary projects, the CS Faculty plans to ensure that there is already sufficient classified image data from the collaborating discipline before starting work on the DL algorithm creation/training portion of the project. 


\section{SE Faculty:}

The work done on the DL algorithm by the CS team requires specific expertise, and the SE Faculty only has a superficial understanding of the process to create/train the DL algorithm. To address the gap in knowledge, SE Faculty believes that leveraging her co-collaborator for her expertise and creating a close and frequent collaboration has been a must. The SE Faculty stated that it is equally important to clearly communicate to the students the desired outcomes of the project, and how the DL algorithm will be implemented in the SE field. By having clear goals and objectives the students will know what type of work the project entails and be able to better gauge their interest in the project, thus reducing the number of students that leave the project due to loss of interest.

\section{Student Perspectives:}

Currently there are two active structural engineering undergraduate students on the project (SE Student $1 \& 2$ ). Each student was interviewed for their perspective on the project, and how it provides a unique experience in their engineering development. A third student (SE Student 3) who had been on the project and moved onto other unrelated research activities with the SE Faculty, was also interviewed. The student interview questions for the SE students is in Appendix B; these questions were modified into an emailed survey form with five-point Likert scale and free response questions for the two undergraduate CS students currently on the project.

The following section of the paper organizes student responses into two areas: (i) how involvement on the research team has impacted their personal engineering development (technical and soft skills, networking, and other professional development), and (ii) project progress and communication.

\section{Research Team Effect on Personal Engineering Development}

A major benefit that the students found in working on this research team were the technical and interpersonal skills they developed through practical engineering experience. Clemson University's Creative Inquiry program facilitated multidisciplinary research on the design of a medical device which engaged students in mechanical engineering, bioengineering, marketing, and nursing departments. These students indicated their participation in the project increased their confidence to enter the workplace [16]. Likewise, students involved in the project discussed in this paper have stated that they are more comfortable and competent with working with academic/industry professionals through this research experience.

\section{SE Student 1:}

SE Student 1 is the first author of this paper and considers this type of research dissemination activity to be highly reflective of the unique opportunities that have been presented to him on this project. SE Student 1's main motivations are to expand his professional network, contribute to research and writing of conference papers/presentations, and to collaborate with industry professionals on a project that has a humanitarian outcome. The research team is addressing a unique shortcoming that exists in the SE field, and SE Student 1 does not think he would gain the same amount of engineering insight in a traditional senior capstone project. In addition, SE Student 1 believes that creating his own deliverables and setting his own schedule has benefited 
the quality of work he has produced and is similar to conditions in industry, which he would not experience within a senior capstone project.

\section{SE Student 2:}

SE Student 2 believes travelling to Mexico City on a reconnaissance mission and working with experienced academics and industry professionals has led to better understanding of concepts from SE coursework. This in-field learning experience allowed her to collect source data for the multidisciplinary project while applying engineering in a way that she feels she never would have had in a capstone project. SE Student 2 has had to develop a higher level of communication skills, set her own work schedule, and contribute to various deliverables led by faculty collaborators from different institutions (from the Mexico reconnaissance). She believes that working on the research team has generated a much higher level of intrinsic responsibility for her work than in her graded upper-division courses or that she anticipates she would have experienced during a traditional senior capstone project. She indicates that the opportunity to develop and apply skills directly relevant to the SE field via this unique multidisciplinary project, especially the seismic performance subspecialty, is valuable to her future career as an engineer.

\section{SE Student 3:}

SE Student 3 joined the research team due to his interest in the technical aspect of the DL algorithm and how it applied to the SE discipline. He felt that working on a research team would diversify his engineering skills and he had interest in the outcome of the project. However, because his tasks mainly consisted of completing a literature review, filtering/tagging image sets, and writing technical documents, he began to lose interest in the project. SE Student 3 stated that he would rather work on the CS team, as he has enjoyed the challenge of past computer programming experiences. His loss of interest in his project work combined with his heavy course load and departure for an upcoming internship were catalysts in leaving the research team. Yet this work and interaction with the SE Faculty was what motivated SE Student 3 to choose his current senior project over a traditional capstone project. In helping redevelop the lab activities for an upper-division structural dynamics course he is able to enhance technical analysis abilities, skills in experimental design and fabrication, computer programming, as well as curriculum development and pedagogy.

\section{CS Student 1:}

CS Student 1 believes that this project has exposed him to engineering in the CS field and he has been able to apply skills learned during CS coursework. Helping to train the DL algorithm has been highly beneficial to his development as a CS engineer since these approaches are becoming extremely commonplace in many industries. Another advantage has been working on project where the outcome is primarily to serve another discipline, which is similar to many software products he will create in his professional career. While traditional senior capstone courses also help to develop engineering skills, CS Student 1 believes that this project is unique since outcomes are directly relevant to industry, as are the opportunities to present at conferences and contribute to conference papers/presentations.

\section{CS Student 2:}

CS Student 2 also indicates that the work done on this project is highly applicable to the CS field, with respect to DL algorithms. He appreciated the latitude the CS Faculty provided to him 
and his CS Student 1 colleague in setting their own tasks and timelines, while having access to guidance when necessary. This opportunity to develop as a self-directed engineer is something he considers important for success in his future career. However, he believes that in the right senior capstone project would have afforded him the same opportunities. The main challenges that he has faced in this multidisciplinary project have been associated with working in the unfamiliar SE field. He notes that overcoming those challenges have been beneficial to his development, as most of his work in the CS field will be with many disciplines where he is novice.

\section{Project Progress and Communication}

A challenge commonly identified by the undergraduate students on this project is the lack of communication between the peer students in the different disciplines. The aforementioned Clemson University project's student responses indicated that communicating issues between disciplines and jointly developing solutions were a valuable part of their research initiative [16]. While the greater communication has been identified as something that could help in the future of this multidisciplinary project, the students do not believe it has been detrimental thus far.

\section{SE Student 1:}

To this point, tasks have been segmented between data gathering on the SE team and using that data to train the DL algorithm on the CS team. SE Student 1 believes that the gap in technical knowledge and lack of clarity on project objectives of the partnering discipline creates challenges in working as efficiently and effectively as possible with that discipline. From his perspective, the process of filtering/tagging images of damaged infrastructure can be improved if the students are aware of: (i) what constitutes bad quality or too few reference points for images used to train the DL algorithm, and (ii) how the DL algorithm is being trained to identify damage in images. Yet, he recognizes that project progress may be hindered by the time it would take to educate the SE team in these two areas. Therefore, SE Student 1 believes that it is extremely important to trust the other team to make clear objectives for their deliverables, and maintains that it is important to have consistent communication and expectations for the whole team.

\section{SE Student 2:}

SE Student 2 also expressed that if she knew more about how the DL algorithm is being developed/trained by the CS team she could produce better quality image sets. Communication between the teams exists at the faculty level. While SE Student 2 does not think that this is an issue for her development as an engineer, she believes that more communication with the students from the partnering discipline might help the progress of the project. Her main motivation for progressing in the project is to apply the data she collected in Mexico. That being said, she does not see the dynamic nature and changing objectives in the project as a negative, so long as there is continual progress and no backtracking. SE Student 2 stated that the small SE team structure is detrimental to progress because of the sheer quantity of image data needed to train the DL algorithm. She also thinks that because the university is a primarily a teaching institution, most students do not understand the amount of work that is required to be an effective research team member. Though she acknowledges that the loss of SE students has interrupted progress in the past, she thinks that the SE team has since stabilized. 


\section{SE Student 3:}

During his time on the research team, SE Student 3 notes that he saw that image sets he had contributed to on the SE team were being used to train the DL algorithm. The damage identification training documents he wrote were kept internally on the SE team. Although, he indicates that he never observed them being used, they were a training resource for SE Students $1 \& 2$ after his departure. He believes that the work he was doing was helping the project progress, but he did not feel that the tasks he completed, in the short term, had any personal learning or professional development benefits to him. His view was that the structural engineering students were grinding through data sets while the CS team was overcoming the real technical challenges. SE Student 3 believes that the project being multidisciplinary was extremely beneficial to the desired outcome; yet, he would have liked to see more work being done together between both teams rather than the delegation of tasks for each team. He believes that the scale of the research team's goal is feasible because it engages multiple disciplines (diverse experts and many players to complete tasks), and will be beneficial to those engineering students interested in the topic.

\section{CS Student 1:}

CS Student 1 acknowledges that he underestimated the project scale and magnitude of work/knowledge required to develop and train a DL algorithm. DL has only just begun to be taught at the authors' institution, so CS Student completed many hours of self-study to develop fundamental proficiency. At the time of the survey, his desired research outcome is to have a "scaffold of a tool" that will be able to classify damaged buildings. His primary challenge has been working on the DL algorithm, rather than collaborating with an unfamiliar field. CS Student 1 believes that interaction solely with his faculty advisor and fellow CS student has not been detrimental to his work, but he would like to meet with the SE team a few times per academic quarter so everyone is abreast of others' work and research outlook. He believes that this crossdiscipline awareness could lead to a more formal iterative process similar to the design cycle in the CS industry. The dynamic objectives that have been associated with the project have not been an issue to CS Student 1's progress, and he has adapted well to the "moving target". CS Student 1 stated that the structure of the team and the students being mostly undergraduates has changed how the project progresses, but he associates that more with how the CS Faculty handles delegating objectives to her students. Overall CS Student 1 thinks that it has been a beneficial project to learn new CS concepts and apply them to a different discipline.

\section{CS Student 2:}

CS Student 2 believes that interaction with the SE students working on the project would help him better understand the goals of the project. Additionally, he indicates that the dynamic nature of the project and changing objectives have had an effect on the progress in the CS team, but he believes that they have adapted well to the changing objectives. CS Student 2 stated that most of the challenge lies with working on a tool that will be used in another discipline. Creating the DL algorithm and the technical skills needed have been a challenge to overcome, but CS Student 2 does not think that progress has been hindered since the resources are available to effectively train the algorithm. In addition, CS Student 2 believes that there is no detriment that the majority of the students are undergraduate students, and disagrees with CS Student 1 that the project is handled differently by the CS Faculty because of their academic status. 


\section{Conclusions:}

The following list of action items, based on faculty and student interviews/surveys, are intended to underscore the benefits of multidisciplinary teamwork and alleviate some of the challenges encountered during the research project described in this paper.

- Seek out multidisciplinary projects and collaborators to innovate in new technical frontiers. Faculty tend to focus their research efforts in their own discipline and collaborate with close colleagues. Today's challenges are ones that often cannot be faced without engaging other disciplines and experts. While it can be daunting to seek out and develop new relationships, from an educational standpoint this study and others [9],[16] indicate that multidisciplinary projects model the scope and diverse problem types students will encounter during an engineering career.

- Couple technical research work with opportunities for students to engage with other academic/industry members and extend their professional network. Student responses indicate that their personal motivation is higher when working on a project that allows them to apply engineering skills and produce tangible outcomes that benefit their field. Faculty should couple applied engineering research with opportunities for students to disseminate their work by preparing papers, presentations, and attending or presenting at professional conferences, or, even conducting field work with external collaborators.

- Student learning gains are just as important as technical deliverables. Faculty should expose students to a topic area that is directly relevant to their future profession. The tasks assigned in this topic area should not be rote activities, but meaningful to the student's continued learning. Faculty should take care that their research group is comprised of students at various levels of expertise to ensure that the faculty themselves are not over-extended trying to meet technical research deliverables and deadlines.

- Be cognizant of dynamic project objectives/deliverables to manage how these affect project progress and student development. Multidisciplinary projects can have a large scope and many research goals. Feedback provided by industry experts to faculty advisors can lead to changing deliverables/objectives over the course of time. This is positive movement; however, care must be taken in managing students to avoid issues with retention due to lost interest or feelings of backtracking. Students that adapt well and are highly motivated by project end goals are less susceptible to negative feelings about change. Both faculty and student responses indicate that the current tasks and objective must be made clear to both teams so that work is synchronized and timely.

- Maintain clear communication to make objectives clear and to keep students engaged with partnering discipline. Even if faculty do not expect significant short-term progress on their respective team, it is critical to have (even brief) weekly meetings regarding updates, any modification in objectives, and intended tasks/deliverables; as recommended by the study conducted by the NSF [17]. Often one team will need the input or assistance of another, and making those items clear in a meeting rather than a series of emails is beneficial. Including the students on a team meeting a few times in an 
academic quarter was recommended in each of the student interviews/surveys conducted for this study. Student responses indicated this would help with cross-discipline understanding, developing strong working relationships, and encouraging student retention.

- Educate students (and faculty) on technical content associated with complementary fields in a multidisciplinary project. A multidisciplinary project often requires a working knowledge of areas that students/faculty have not been exposed to in their own field. It is of their benefit to either take a formal course or engage in peer-teaching during project meetings to learn about the complementary project discipline(s). This background is imperative to ensure that all research team members are producing quality products which the collaborating discipline can use to progress the overall research aims.

\section{Acknowledgements:}

The authors would like to thank the collaborating faculty and students on this multidisciplinary project for sharing their experiences through surveys and interviews. They would also like to thank California Polytechnic State University - San Luis Obispo for their generous funding of the discussed technical research work via CPConnect and Research, Scholarly, and Creative Activities (RCSA) Grant Programs.

\section{References:}

[1] "The Earthquake Engineering Online Archive NISEE e-library,” nisee.berkeley.edu, 2016. [Online]. Available: https://nisee.berkeley.edu/elibrary/

[2] "Learning from Earthquakes Reconnaissance Archive," eeri.org, 2016. [Online]. Available: https://www.eeri.org/projects/learning-from-earthquakes-lfe/lfe-reconnaissance-archive/

[3] "Reconnaissance Reports," geerassociation.org, 2015. [Online]. Available: http://www.geerassociation.org/reconnaissance-reports

[4] "Resources: Databases," datacenterhub.org, 2014. [Online]. Available: https://datacenterhub.org/resources/databases

[5] A. Behrouzi, M. Pantoja. "Photo Tagging Tool for Post-Earthquake Structural Damage Identification". 11th US National Conference on Earthquake Engineering (11NCEE), Los Angeles, CA. June 25-29 2018.

[6] B. Patterson, G. Leone, M. Pantoja, and A. Behrouzi. "Deep Learning for Automated Image Classification of Seismic Damage to Built Infrastructure". 11NCEE, Los Angeles, CA. June 25-29 2018.

[7] A. Behrouzi, M. Pantoja. "Software Toolset to Enable Image Classification of Earthquake Damage to Above-Ground Infrastructure." 16th European Conference on Earthquake Engineering, Thessaloniki, Greece. June 18-21, 2018.

[8] L. Fie-Fie, K. Li, "ImageNet," image-net.org, 2016. [Online]. Available: http://imagenet.org/index

[9] R. Munasinghe, "Multidisciplinary Research Projects for Engineering Students", in 2005 ASEE Annual Conference, Portland, Oregon. 
[10] O. Despo, S. Yeung, and J. Jopling, "BURNED: Towards Efficient and Accurate Burn Prognosis Using Deep Learning". 2017. [Online]. Available: http://cs231n.stanford.edu/reports/2017/pdfs/507.pdf [Accessed Feb 3, 2018]

[11] J. A. Calderon-Martinez and P. Campoy-Cervera, "An Application of Convolutional Neural Networks for Automatic Inspection," in 2006 IEEE Conference on Cybernetics and Intelligent Systems, Bangkok, p. 1-6, 2006.

[12] P. Juell and R. Marsh, "A Hierarchical Neural Network for Human Face Detection", Pattern Recognition, vol. 29, no. 5, p. 781-787, 1996.

[13] Z. Zhai, T. Kijewski-Correa, D. Hachen and G. Madey, "Haiti earthquake photo tagging: Lessons on crowdsourcing in-depth image classifications," in Seventh International Conference on Digital Information Management (ICDIM 2012), Macau, p. 357-364, 2012.

[14] F. Dai, S. Dong, V. Kamat, and M. Lu, "Photogrammetry Assisted Measurement of Interstory Drift for Rapid Post-disaster Building Damage Reconnaissance", in Journal of Nondestructive Evaluation, vol. 30, p. 201-212, 2011.

[15] Y. Chul, S.J. Dyke, and J. Ramirez, "Visual Data Classification in Post-Event Building Reconnaissance", Engineering Structures, vol. 155, p. 16-24, 2018.

[16] F. Sahin and W. Walter, "Multidisciplinary Microrobotics Teaching Activities in Engineering Education", in 2003 ASEE Annual Conference, Nashville, Tennessee.

[17] J.N. Cummings and S. Kiesler, "Collaborative Research Across Disciplinary and Organizational Boundaries,” Social Studies of Science, vol. 35, p 703-722, Oct 2005.

[18] R. K. Anderson, T. Schweisinger, and B.J. Speziale, "Strengthening Undergraduate Career Preparedness through Multidisciplinary Research Projects", in 2015 ASEE Annual Conference, Seattle, Washington. 


\section{Appendix A: Faculty Interview Questions}

\section{Cross-discipline Understanding and Communication}

1. This multidisciplinary project brings together fields (structural engineering and computer science) that would not typically intersect in a student's academic training. How does this project expose your students to applied engineering across multiple disciplines, versus traditional upper-division coursework and senior projects?

2. One of this project's ultimate goals is to produce a structural engineering tool. What challenges and learning gains have you experienced working on a project where you are not a subject area expert? How has it differed from research projects that are solely within your discipline?

\section{Project Progress and Outcomes}

3. What are your desired project outcomes for both you and your students?

4. The specific research goals of this project have shifted over time, and the structural engineering team has had to change course multiple occasions. With respect to the work in your discipline, how have you perceived the dynamic nature of the project?

5. How has it affected the management of your team? How have you seen your students adapt to a "moving target"?

\section{Miscellaneous}

6. This project's research team consists of mostly undergraduate students between both disciplines. How have you approached assigning tasks and delegating responsibility to undergraduate students versus graduate students? 


\section{Appendix B: Student Survey Questions}

Note: [Likert] and [Free Response] designations indicate the question type posed to CS students in their email survey. All SE Students answered questions via interview as Free Response.

Questions were either free response or used the Likert scale seen below

1. Strongly agree

2. Agree

3. Neutral

4. Disagree

5. Strongly disagree

\section{Cross-discipline Understanding and Communication}

1. This project is multidisciplinary across two unique engineering disciplines (computer science and structural engineering). Do you think this project exposed you to applied engineering in your discipline that you do not think you would normally see in traditional coursework? [Likert]

2. Has it been challenging in collaborating with a structural engineering team? [Likert]

3. Do you think working with another team outside your discipline will help you in the future to become a better engineer in your discipline? [Likert]

\section{Project Progress and Outcomes}

4. What are your desired project outcomes? Thus far, what are your primary concerns about the progress in reaching those desired outcomes?

[Free Response]

5. The specific research goals of this project have shifted over time, and the structural engineering team has had to change course multiple occasions. Do you think you adapt well to the "moving target"?

[Likert]

\section{Miscellaneous}

6. This project's research team consists of mostly undergraduate students between both disciplines. Do you think this project benefits from the team being mostly undergraduate students?

[Likert]

7. Is there anything else you would like to share with me about the project that you would like to share with other students? Your excitement, what you see for the future, lessons learned, etc.?

[Free Response] 